ESAIM: PROCEEDINGS AND SURVEYS, September 2014, Vol. 45, p. 255-264

J.-S. Dhersin, Editor

\title{
"PROGRESSER EN GROUPE" (PEG): UNE MÉTHODE PÉDAGOGIQUE GLOBALE BASÉE SUR LE TRAVAIL EN PETITS GROUPES
}

\author{
Christophe RABUt ${ }^{1}$
}

\begin{abstract}
The main point of the method is the following one: replace lectures by team working (a team is made of four students). Two ways are used for that: First way, "beforehand material studying": as a home work the student have to study a document ("from page so and so to page so and so"), then, when in classroom, the students talk within teams in order to solve the difficulties they have identified, help each others, ask for help to the professor... Second way, "problemized lecture": the lecture is written on the form of a problem, to be solved by the teams, possibly after individual homework on it. As for exercises and problems, they too are studied in teams, with or without previous homework. "Synthesizing and structuring lectures" then complete the package, often introduced by a "problem for positioning and motivation". The whole package is an innovating and very efficient procedure for which advantages and constraints are described in this paper."

Résumé. Le cœur de la méthode est celui-ci : nous remplaçons les cours magistraux par du travail en "équipes" de quatre étudiants. Deux stratégies différentes sont utilisées pour cela. Ou bien le "cours préparé à l'avance" : en travail personnel les étudiants étudient un document ("de la page tant à la page tant"), puis, en séance, regroupés par équipes de quatre, ils échangent sur leurs difficultés, s'aident mutuellement, demandent éventuellement à l'enseignant de les aider. Ou bien, le "cours problématisé" : le cours est transformé en problème, que les étudiants doivent résoudre en équipes, éventuellement après une préparation en travail personnel. Les exercices et problèmes sont aussi traités en équipes, avec ou sans préparation personnelle. Des "cours de synthèse et structuration" viennent compléter le dispositif, par ailleurs en général introduit par un "problème de positionnement-motivation". Cet ensemble de mesures constitue un dispositif innovant et très efficace, dont les avantages et les contraintes sont détaillés dans cet article.
\end{abstract}

\section{INTRODUCTION : INSATISFACTION CONCERNANT LES COURS MAGISTRAUX}

Soyons francs : nous sommes souvent insatisfaits de l'efficacité de nos cours magistraux. "Les étudiants ne sont pas assez attentifs", "ils ne suivent pas", "ils ne travaillent pas le cours avant les TD"... de sorte que souvent les TD reprennent une partie du cours, soit explicitement (rappels préalables !), soit plus ou moins implicitement, à l'occasion du corrigé des exercices... ce qui ne peut qu'encourager les étudiants à ne pas travailler le cours. Cette insatisfaction est d'ailleurs partagée par les étudiants, puisque le taux de présence est souvent inférieur à ce que nous voudrions qu'il soit... Je me trompe ? Qu'en est-il pour vous ? Je n'insiste pas en détail sur les raisons de ce qui me semble être un échec méthodologique, mais disons rapidement qu'il est impossible de s'adapter aux besoins (rythme, type de motivation) de chaque étudiant dans la salle, et que

${ }^{1}$ christophe.rabut@insa-toulouse.fr. INSA, Département de Génie Mathématique et Modélisation, 31077 Toulouse Cedex 4

(C) EDP Sciences, SMAI 2014 
ceux-ci sont rarement vraiment motivés par un cours "théorique" dont ils ne voient pas immédiatement l'utilité, qu'enfin l'accumulation de cours dans une même journée rend souvent les étudiants passifs, voire blasés...

Bien sûr, nous faisons tout notre possible pour "limiter les dégâts" : cours aussi vivants que possible, voire humour ou anecdotes, éléments de motivation historiques ou d'application... mais force est de constater que, malgré tous nos efforts, "cela ne passe pas bien".

Alors que faire? Supprimer les cours? Pas vraiment, car justement nous pensons tous que notre enseignement ne doit pas, loin s'en faut, se limiter aux savoir-faire, et nous tenons à ce que les étudiants travaillent et assimilent ce que nous leur présentons en cours...

Face à cela, de nombreux enseignants et de nombreuses institutions se sont penchés sur le problème, et se tournent vers les "pédagogies actives" (comprenez en général les pédagogies impliquant du travail de groupe), et notamment l'Apprentissage par Problèmes et par Projets (APP), ("Problem Based Learning" ou PBL if you prefer speaking english ; voir par exemple [2] pour une présentation de la méthode, et [3] pour son implantation à Louvain-la-Neuve).

Inspiré par cela, mais insatisfait par certains points (les étudiants sont trop souvent, je pense, livrés à euxmêmes), j'ai progressivement mis au point une méthode d'enseignement, ou plutôt une méthode de travail avec les étudiants, que je présente ici. Soyons clairs, bien que maintenant éprouvée et vivement appréciée par ceux qui ont décidé de franchir le pas (voilà maintenant cinq ans qu'elle est formalisée et pratiquée de façon conforme à ce qui est présenté ici), son utilisation dans son intégralité est encore tout à fait marginale. Trois enseignants l'appliquent ou l'ont appliquée sur l'INSA de Toulouse (en mécanique, mécanique des fluides, et en mathématiques appliquées) l'un d'eux étant maintenant retraité. Deux professeurs de maths de lycée (l'une en terminale S à Toulouse, l'autre en Seconde à Grenoble) l'appliquent de façon systématique, avec quelques adaptations liées aux spécificités du lycée. Des professeurs de mathématiques de l'École Polytechnique de Louvain (EPL, à Louvain-la-Neuve) font quelque chose d'assez proche, mais moins directif sous le nom d'Apprentissage par Problèmes. Bien que cela soit insuffisant pour en tirer une règle générale, notre expérience (et la satisfaction combinée des enseignants et des étudiants) suffit pour dire à quel point cela nous parait plus efficace et plus intéressant que la méthode des cours magistraux préalables, que nous avons tous largement pratiquée dans le passé. Nous sommes maintenant dans la phase de diffusion de l'information sur cette méthode, d'où la Table Ronde au colloque de Seignosse et cet article...

Examinons tout de suite deux solutions proposées pour "résoudre" ce problème. Toutes deux sont basées sur le travail du cours en équipe (nous appellerons ici "équipe" un petit groupe d'étudiants, idéalement groupes de quatre étudiants). Nous analyserons ensuite certains avantages et certaines contraintes.

\section{ETUde Du COURS EN ÉQUIPE}

\subsection{Le "cours étudié à l'avance"}

Les étudiants doivent arriver en séance en ayant étudié une partie précise d'un document ("page tant à tant de tel livre, de tel polycopié"... ). Pendant ce travail préalable, ils doivent identifier les difficultés et les points importants. En séance, les étudiants d'une même équipe confrontent leurs interrogations, leurs difficultés, leurs incompréhensions, ainsi que les points qu'ils considèrent acquis lors de leur travail individuel. Ils s'expliquent mutuellement les points compris par certains et pas par d'autres, résolvent (ou tentent de résoudre) ensemble les difficultés communes, approfondissent les points clef...

Lorsqu'un point reste non résolu, l'équipe se met d'accord pour faire appel à l'enseignant, formule une question à laquelle l'enseignant répond. A noter que le type de réponse dépend beaucoup de l'à-propos pédagogique de l'enseignant. Ce peut être une réponse très courte, validant en quelque sorte ce qui a été dit, ou une réponse longue, prenant le temps même d'aller au-delà de la question proprement dite. C'est souvent aussi une réponse presque incomplète, mettant l'équipe sur la bonne voie et lui laissant le soin de progresser par elle-même dans la direction indiquée. De cette confrontation naît une meilleure compréhension et assimilation du cours. Par ailleurs le cours est travaillé régulièrement, au fur et à mesure, et non en fin de séquence, quand vient l'examen... 


\section{Répondons tout de suite à certaines questions à ce propos.}

Les étudiants font-ils le travail personnel ? Oui, la plupart du temps. En effet d'une part ils se rendent vite compte que c'est leur intérêt, puisque, s'ils n'ont pas compris tel ou tel point, l'équipe pourra les aider à résoudre cette question, alors que s'ils n'ont rien fait, il leur faudra bien étudier le cours plus tard (celui-ci ne sera pas présenté par l'enseignant), et ils n'auront plus l'équipe pour les aider. De plus, selon une remarque plusieurs fois exprimée par les étudiants, ceux-ci ne souhaitent pas "être le boulet de l'équipe"... il y a donc, en général, une certaine émulation au sein des équipes.

Que faites-vous si un étudiant n'a pas fait le travail préparatoire? Pas de problème dans la mesure où c'est exceptionnel (en nombre d'étudiants concernés et en nombre de fois où cela arrive) : l'équipe absorbe cette perturbation. Si nécessaire, on peut remixer les équipes en regroupant les étudiants n'ayant pas fait le travail préparatoire... ils perçoivent vite l'intérêt de faire cette préparation de la séance !

Cette méthode prend-elle plus de temps qu'un cours magistral traditionnel ? L'expérience montre que non. Évidemment, il faut tenir le temps, et faire attention de ne pas laisser passer plus de temps que prévu... mais notons qu'un des avantages de cette façon de procéder est que cela permet à chaque étudiant d'avancer à son rythme, en particulier au niveau de son travail personnel. Un étudiant plus lent, ou moins avancé, pourra compenser en passant plus de temps à la partie de préparation, et ainsi se trouver à même d'échanger avec ses coéquipiers... alors que lors d'un cours magistral, l'étudiant trop lent ne peut plus suivre au bout d'un certain temps, il décroche, perd donc son temps (et son moral!).

\subsection{Le cours transformé en problème}

Le cours est transformé en problème, posé aux équipes. Les théorèmes sont démontrés par des questions successives, puis synthétisés sous forme explicite. Les équipes cherchent à résoudre le problème, les coéquipiers acquièrent les notions par le débat et les échanges que suscitent les difficultés... Un exemple est donné en annexe, utilisé en 2ème année d'école d'ingénieur (analyse numérique, discrétisations de la dérivée, erreur de méthode et erreur d'arrondi).

Là encore, on utilise pleinement la force de l'équipe : seuls, ils ne pourraient dépasser l'obstacle que constituent les notions nouvelles mises en œuvre. L'effet "brainstorming" joue à plein, la collaboration et l'effet d'entrainement atténuent grandement la difficulté.

Est-ce que cela prend plus de temps qu'un cours magistral traditionnel ? OUI et NON. Oui, car, c'est clair, il est bien plus rapide de présenter une notion, de démontrer un théorème, que de demander aux étudiants d'avancer eux-mêmes dans la démonstration (même si, bien sûr, ils sont soigneusement guidés). Il faut bien deux fois plus de temps pour arriver au même point. Cependant les étudiants ont effectivement travaillé sur le sujet, ont argumenté sur la question, ont bien mieux compris et intégré les détails de la partie étudiée, l'importance de telle ou telle hypothèse. Du point de vue du temps proprement dit, on y gagne sur plusieurs tableaux à la fois : meilleure compréhension et assimilation, de sorte qu'il est beaucoup moins nécessaire, par la suite, de revenir dessus ; les étudiants font en même temps du cours et des problèmes/exercices... de sorte qu'il est possible de passer moins de temps sur les exercices.

\section{LE DISPOSITIF D'ACCOMPAGNEMENT}

Plusieurs pratiques complètent et renforcent l'efficacité de l'étude du cours en équipe : le cours de synthèsestructuration, le problème de positionnement-motivation, les exercices faits à la maison et corrigés éventuellement en équipe, les problèmes traités en équipe.

\subsection{Le cours de synthèse-structuration}

C'est un complément essentiel aux cours travaillés en équipe. Il faut structurer les connaissances acquises en travail personnel et en équipe. Pour cela, de temps en temps (ma pratique est d'une séance toutes les quatre ou cinq séances), un cours magistral a pour but de recadrer les choses, de commenter ce qui a été fait, peut-être de mettre mieux en évidence tel ou tel point essentiel, de compléter le travail des équipes, voire d'aller plus loin 
dans telle ou telle notion abordée. Pas question de reprendre ce qui a été fait en équipe (ce serait le meilleur moyen d'inciter les équipes à "se la jouer cool" !), ces cours magistraux s'appuient sur ce qui a été fait, mettent les notions en perspective, les relient à d'autres notions, quelquefois les prolongent.

L'image que je préfère est celle du vélo : pendant le travail du cours en équipe, les étudiants sont, essentiellement, "le nez dans le guidon", examinant les choses en détail, mais sans vraie liaison avec le reste de la matière. Le but du cours de synthèse-structuration est alors de leur faire lever la tête pour regarder le paysage, voir où ils sont par rapport à ce qu'ils connaissent déjà, voir où ils vont dans ce contexte, articuler les notions avec d'autres notions qu'ils pourraient croire sans point commun. J'utilise aussi l'image du puzzle : le cours de synthèse-structuration assemble les pièces du puzzle étudiées en travail personnel et en équipe.

Clairement il s'agit là d'un cours magistral, qui peut être fait devant des grands groupes, à l'image de nos cours magistraux habituels. Mais le but et la façon de le faire en sont très différents, comme l'attitude des étudiants vis-à-vis de ce cours. En effet, pour l'essentiel il ne présente pas de choses nouvelles, mais il s'appuie sur ce que les étudiants savent déjà pour articuler ces notions entre elles. Ce qui y est dit est en lien avec les préoccupations des étudiants, qui en voient tout de suite l'intérêt et l'importance. Les étudiants ne s'y trompent d'ailleurs pas : le taux de présence, la qualité d'écoute sont sans commune mesure avec ceux d'un cours magistral classique.

\subsection{Le problème de positionnement-motivation}

Pour souder les équipes, leur permettre de se rendre compte de l'intérêt du travail en équipe, et motiver les étudiants pour le travail personnel préalable, un problème de "positionnement-motivation" est le bienvenu en début d'enseignement.

On ne demande pas aux étudiants de résoudre un problème complet, mais on leur demande plutôt de trouver une démarche de travail. Bref on leur demande "comment ils feraient pour résoudre le problème" plutôt que de leur demander de le résoudre en détail. A ce stade, il ne s'agit pas de résoudre complètement le problème posé, mais de suggérer (enseignant) puis de préciser (étudiants) plusieurs démarches de résolution, qui justement seront ensuite étudiées et approfondies en cours. A noter que l'effet "brainstorming" permet de poser des problèmes qui sont de vrais défis. Dans certains cas ce problème est utilisé comme "fil rouge" de l'enseignement : périodiquement (par exemple lors de certains cours de synthèse et structuration) on reprend le thème du problème en expliquant en quoi on a avancé dans la résolution potentielle du problème. Ce problème peut être plus ou moins important, il est en général basé sur une application ou une utilisation d'un ou de plusieurs points importants du cours.

\subsection{Les exercices et problèmes}

Il est bon ici d'utiliser toute la force du travail en équipe, mais donc, en particulier, de ne pas poser des exercices d'application immédiate à résoudre entièrement en équipe. La solution semble être celle-ci : distinguer les exercices d'application immédiate du cours, qui n'ont pas a priori besoin d'être traités en équipe, des problèmes à initiative, qui sont au contraire tout à fait appropriés pour un travail en équipe.

Les exercices d'application immédiate du cours : ils sont de préférence posés en travail personnel à faire à la maison, les étudiants d'une même équipe assurant entre eux le "corrigé", c'est-à-dire la comparaison rapide de leurs résultats et/ou méthode exacte de résolution, et ne discutent vraiment de l'exercice que s'il y a des différences importantes au sein de l'équipe.

Par contre, pour les problèmes plus importants, en particulier pour ceux qui sont posés de façon à laisser de l'initiative, aux étudiants, ou ceux qui présentent plusieurs méthodes de résolution, seule une prise de contact est faite en travail personnel préalable, puis, en séance, les équipes doivent trouver une stratégie de résolution, et mettre en œuvre cette stratégie. Les effets "brainstorming" et "discussion-contestation-comparaison des méthodes de résolution" peuvent alors jouer à plein, pour le plus grand bénéfice de la qualité de compréhension et d'assimilation des notions étudiées. En général, ces problèmes se déroulent en trois phases. La première phase, au niveau de l'équipe, consiste à définir une (ou plusieurs) méthode(s) de résolution, à comparer éventuellement des méthodes suggérées par différents coéquipiers. Dans la seconde phase les différents coéquipiers effectuent 
chacun tout ou partie des calculs définis dans la première phase (ils peuvent s'aider, se répartir la tâche, voire utiliser certains une méthode, d'autres une autre méthode). La troisième phase, de nouveau travail d'équipe, consiste à comparer les résultats obtenus et à tirer les conclusions pour achever le travail.

\section{4. Évaluation}

Les moyens d'évaluation restent traditionnels. Il n'y a pas d'évaluation du travail de groupe, et aucune production n'est demandée aux équipes. Le fait de travailler en équipe est une méthode d'acquisition et d'assimilation, mais pas une compétence qui doit être chiffrée, valorisée en tant que telle.

\subsection{Bilan}

L'ensemble de ces techniques constitue un ensemble cohérent et efficace, une méthode d'enseignement que nous appelons à l'INSA de Toulouse "Progresser En Groupe" (PEG). Il est bien sûr possible d'utiliser certains points sans utiliser les autres, mais il faut alors veiller à ce que la quantité de travail en équipe et la qualité de l'exigence concernant ce travail, soient suffisantes, car il y a une sorte de "masse critique" pour que ce travail en équipe donne toute sa puissance et son efficacité.

\section{Analyse, remarques et commentaires}

\subsection{Intérêt du travail en équipe}

Un groupe, c'est magique. Faire travailler les étudiants en petits groupes (idéalement équipes de quatre étudiants) présente beaucoup, beaucoup d'avantages. Essayons d'en lister quelques-uns.

Tout d'abord, on retient beaucoup mieux ce que l'on a discuté, ce pour quoi on a cherché des arguments, et/ou écouté les arguments des autres suite à une intervention de notre part ; certains affirment même [1] que l'on retient environ $10 \%$ de ce que l'on lit, $20 \%$ de ce que l'on entend, $70 \%$ de ce que l'on dit (dans un débat, une discussion)...

Formuler une question, intervenir dans une discussion, oblige à faire un peu le tour de la question, et aide à prendre un peu de recul, à choisir les bons mots pour être compris. Cela aide donc à s'exprimer correctement, dans un premier temps par oral, qui peut être une étape pour une meilleure qualité de rédaction écrite.

On n'a vraiment bien compris quelque chose que lorsqu'on l'a (correctement) expliqué... De sorte qu'un membre d'une équipe demande à un autre membre de l'équipe de lui expliquer un point (théorème, démonstration, application... ) qu'il ne comprend pas, les deux étudiants y gagnent (ainsi que les autres membres de l'équipe, qui écoutent et éventuellement participent à la réponse ou aux questions), celui qui explique car cela l'aide à clarifier, et celui qui a posé la question... écoute attentivement, et n'hésite pas à reformuler sa question si la réponse ne lui a pas apporté les éclaircissements espérés.

L'effet "brainstorming" (je préfère dire "jaillissement d'idées") fonctionne très bien dans le domaine scientifique (nous le savons bien, c'est pour cela que nous faisons des réunions d'équipe de recherche...). C'est-à-dire qu'un début d'idée, encore mal formulée, peut être repris par un autre étudiant de l'équipe, pour la formuler mieux, la prolonger, ou même peut donner naissance à une autre idée, qui peut encore être reprise, amplifiée, contestée par un autre... De sorte qu'une équipe peut aborder et résoudre des questions qu'aucun membre de l'équipe n'aurait été capable de résoudre seul. On peut donc poser à des équipes des problèmes que l'on ne peut pas poser à des étudiants travaillant seuls.

A noter que ce point est fondamental, car, grâce au fonctionnement par équipe, nous pourrons poser des types de problèmes que nous ne pourrions poser aux mêmes individus travaillant pour l'essentiel isolément. Grâce à cela, les étudiants peuvent aborder des notions nouvelles en équipes (cours préparé à l'avance, cours problématisé). Ils peuvent aussi résoudre des problèmes à initiatives, ou des problèmes admettant plusieurs solutions, pour lesquels la discussion d'équipe permet d'aborder de façon beaucoup plus approfondie les problèmes posés. 
"L'enseignant répond aux questions des équipes" : l'équipe doit, pour poser une question, se mettre d'accord sur le fait qu'elle n'a pas réussi à résoudre le problème, et sur la formulation de la question. Voilà un excellent exercice (qui de plus permet quelquefois à l'équipe de se rendre compte qu'elle peut résoudre cette question !).

Oui, un groupe, c'est magique, cela permet de faire plus et mieux...

\subsection{Rythme de travail}

Le travail est fait régulièrement (pas seulement à la fin, pour les examens !), les notions sont acquises au fur et à mesure. La plupart du temps les étudiants ont correctement assimilé l'essentiel des chapitres précédents lorsqu'ils étudient les chapitres suivants... Voilà qui est très confortable, pour l'enseignant comme pour les étudiants... et qui permet un gain de temps appréciable car les rappels sont beaucoup moins nombreux.

L'enseignant domine l'avancement des travaux à peu près de la même façon qu'avec une méthode traditionnelle. Il se rend cependant mieux compte si telle notion nécessite plus ou moins de temps pour être correctement étudiée par les étudiants.

\subsection{Temps de travail et efficacité}

Le travail personnel est assez bien cadré et les étudiants ne partent pas dans des directions inutiles. La quantité de travail nécessaire pour obtenir certains acquis est en général du même ordre que lors d'une pédagogie traditionnelle, pour un acquis bien plus en profondeur, et avec davantage de recul. On obtient donc une meilleure efficacité dans le travail qu'avec une pédagogie traditionnelle, ainsi qu'avec la plupart des pédagogies actives.

Le problème de positionnement-motivation nécessite du temps, et n'a pas son équivalent dans l'enseignement traditionnel. C'est cependant un investissement très intéressant et très rentable, car les étudiants sont dès le départ impliqués dans la matière qu'ils vont étudier. C'est plus coûteux en temps, mais beaucoup plus efficace que la présentation par l'enseignant du problème et des directions de solutions qui vont ensuite être étudiées... et bien sûr est autrement mieux que la présentation a posteriori d'une application-utilisation des notions étudiées.

\subsection{Meilleure acquisition}

Bien meilleure mémorisation grâce aux débats entre étudiants (on mémorise beaucoup mieux ce dont on a discuté, ce sur quoi on a argumenté, voir [1]). Dans leur travail individuel, les étudiants apprennent à préciser quels sont les points qui leur font difficulté et à formuler clairement des questions à ce sujet. Cette méthode les aide à prendre du recul par rapport à leur travail et à la matière étudiée. L'enseignant perçoit mieux les difficultés que rencontrent les étudiants (observation des équipes, mais aussi "questions de l'équipe", qui correspondent aux points de résistance), ce qui peut l'aider à mieux insister sur ces points lors des cours de synthèse-structuration, voire à re-rédiger certains points du polycopié.

\subsection{Meilleure auto-évaluation}

Par les discussions au sein de l'équipe, les étudiants se rendent mieux compte de ce qu'ils ont compris et de ce qu'il leur faut encore travailler, et ceci au fur et à mesure que se déroule l'enseignement.

\subsection{Phase de travail préalable}

Cette phase est extrêmement utile et extrêmement efficace (bien plus que le travail qui suit un cours magistral). Elle est faite sérieusement car l'étudiant voit où il va et est motivé grâce au problème de positionnementmotivation, enfin car il sait que le cours ne sera pas exposé en cours magistral. Rapidement, il se rend compte de l'efficacité de cette méthode et de l'intérêt de faire le travail préparatoire du cours. Préparer les questions à poser à l'équipe, risquer de devoir répondre aux questions d'autres membres de l'équipe l'incitent à faire sérieusement ce travail. La discussion en équipe lui permet de se rendre compte s'il avait bien compris les points difficiles. Devoir reformuler pour expliquer un point du cours (ou écouter la reformulation faite par un autre étudiant) est un facteur important de compréhension et d'assimilation. 


\subsection{Répartition des tâches}

Cette façon de travailler en équipe n'incite pas à la répartition des tâches (et des compétences). En effet l'équipe n'a pas de production à rendre (poster, exposé, compte-rendu d'équipe...), de sorte qu'il n'y a pas de confusion sur le but du travail en équipe.

\subsection{Solidarité entre étudiants, capacité à dialoguer}

Lors du travail en équipe les étudiants apprennent à écouter l'autre, à répondre à ses questions, en se plaçant dans le registre de l'autre, bref à accepter la différence, à dialoguer. Apprentissage aussi de la solidarité entre étudiants, à aider les autres, mais aussi à n'avoir pas de scrupules à demander de l'aide. Enfin cela aide les étudiants à se rendre compte qu'ils ne sont pas les seuls à avoir des difficultés, quelquefois aussi à se rendre compte qu'ils croyaient avoir compris mais qu'ils avaient sauté sur une difficulté...

Les étudiants analysent ce que l'équipe leur a apporté (puisque grâce à l'équipe ils résolvent des problèmes sur lesquels ils butaient, et que ceci est clairement identifié). Ils se rendent compte si leur travail personnel a été superficiel ou non (puisque lorsque d'autres étudiants leur posent des questions, ils voient s'ils avaient perçu la difficulté, s'ils savent y répondre).

Plusieurs effets collatéraux sont importants : apprendre à s'écouter, à répondre dans le registre de son interlocuteur, à s'exprimer correctement...

\subsection{Rôle de l'enseignant}

Les questions des équipes sont bien sûr les bienvenues. L'enseignant veille cependant à ce qu'il s'agisse bien d'une question sur laquelle l'ensemble de l'équipe a réfléchi avant de solliciter son aide. Pour cela, il peut, par exemple, choisir l'étudiant qui pose la question au nom de l'équipe, et demander à l'équipe si elle est d'accord sur sa formulation, avant d'aborder le sujet avec l'équipe. Le type de réponse (re-questionnement, détail technique, contre-exemple...) dépend bien sûr de la question posée et de l'à-propos pédagogique de l'enseignant.

Bien sûr le rôle de l'enseignant est profondément modifié. De "personne qui sait et transmet son savoir", il devient une aide à la construction du savoir, et est bien perçu comme tel. Ce qui change tout dans la vision que l'enseignant a de son rôle, et que l'étudiant a du sien, mais aussi le rapport enseignant-étudiant. Loin de perdre sa qualité d'enseignant en n'introduisant plus les notions au tableau, il est pleinement dans son rôle quand il fait des cours de synthèse-structuration (plus passionnants à concevoir que de dérouler des théorèmes !), quand il aide les équipes à résoudre leurs difficultés... et quand il rédige un document adapté à un travail autonome.

\subsection{Initiation à la recherche}

Cela ne vous aura pas échappé : ce travail en équipe, travail préparatoire autonome d'acquisition de notions, suivi d'un travail en équipe, l'utilisation de l'équipe en "brainstorming" pour trouver une méthode de résolution, confrontation des résultats en équipe... voilà des démarches qui sont bien proches des nôtres en recherche. Au moment où nous devons faire une certaine initiation la recherche, il me semble que PEG est un très bon moyen dans cette direction...

\subsection{Qu'en pensent les étudiants ?}

Voilà six ou sept ans que "PEG" est mis en œuvre sous cette forme (il a fallu environ quatre ans pour la mettre au point), et les résultats des bilans d'enseignement montrent que les étudiants, dans leur quasi-totalité, apprécient beaucoup de travailler ainsi. Ils estiment en général que cela ne nécessite pas plus de travail que par une méthode traditionnelle, qu'ils ont beaucoup plus de plaisir et de motivation, qu'enfin la contrainte de travailler régulièrement leur est particulièrement bénéfique. Le fait de mieux percevoir leurs progrès, mais aussi leurs difficultés contribue à leur motivation. 


\subsection{Comment mettre cela en œuvre dans nos enseignements ?}

Souplesse Facile à mettre en œuvre, cette méthode est facilement mixable avec une méthode plus traditionnelle, que ce soit au sein d'un même enseignement ou en "cohabitant" avec d'autres enseignements plus traditionnels.

A quel niveau l'appliquer ? Tous les niveaux universitaires sont possibles. Il est clair en tous cas qu'il est possible de l'utiliser dès le niveau L1. Je pense même que ce serait une bonne façon d'inciter les étudiants à modifier leurs méthodes de travail par rapport à ce qu'ils faisaient au lycée, que cela contribuerait ainsi à un changement de mentalité à l'arrivée en université, et serait de plus un élément de lutte contre l'échec des jeunes étudiants. Bien sûr cette méthode est tout à fait utilisable pour la plupart des matières.

\subsection{Quelles en sont les difficultés ?}

Tout d'abord changer! Oui, changer nos habitudes, nooos pratiques, nos mentalités, ce n'est pas facile. La plupart des enseignants à qui nous parlons de cette façon de travailler se montrent intéressés... mais hésitent à faire le pas ("on verra l'an prochain..."). De plus on craint souvent que cela demande du temps (en fait pas tant que cela), et l'ensemble de nos activités nous presse à tous les niveaux... pas facile! Mais, c'est sûr, passionnant!

Deux difficultés techniques, et une difficulté administrative, essentiellement :

1. La nécessité de mettre à la disposition des étudiants un document qui leur permette de travailler de façon autonome. Livre ou polycopié, à chacun de voir en fonction de sa sensibilité et des spécificités de l'enseignement concerné. Il me semble que les livres sont souvent rédigés de façon peu conviviale et se prêtent mal à une première approche en autonomie, surtout lorsque les étudiants ne sont pas habitués à cette façon de travailler. Je préconise, au moins pour le ou les premiers enseignements effectués en cours préparés en autonomie et approfondis en équipe, de rédiger un polycopié en pensant aux différentes digressions que nous mettons en cours magistral pour soutenir l'intérêt et l'attention des étudiants, et de les mettre dans le polycopié.

2. Par ailleurs, il n'est pas évident, lors de la mise en place de cette méthode pour un enseignement, de savoir exactement l'agencement des séances (en particulier quand placer les cours de synthèse-structuration), ni le temps nécessaire pour telle ou telle partie. Le plus simple est de se baser sur le cadencement des séances faites sous forme traditionnelle, et de reconduire ainsi le rythme d'avancement.

3. Bien sûr, il faut transformer certaines séances de cours en amphi par des séances de travail de cours en équipe... d'où un certain coût, à négocier avec les autorités compétentes... une certaine sensibilité sur la lutte contre l'échec, sur les "méthodes actives", devrait aider à convaincre vos responsables.

\subsection{Peut-on ne faire que la partie "cours problématisé" ?}

Oui. C'est même une bonne façon pour commencer "en douceur". Transformer un cours en problème est beaucoup plus simple que je ne pensais. On peut aussi tout à fait transformer le début d'un chapitre en problème, puis prolonger le problème sous la forme d'un cours de synthèse-structuration. Les étudiants s'accrocheront alors beaucoup plus au cours car ils en auront en quelque sorte créé eux-mêmes les bases et apprécieront de voir les conséquences de ce sur quoi ils ont travaillé.

\section{Conclusion}

Une chose est sûre : quelles qu'en soient les raisons, un cours magistral préalable, présentant les notions nouvelles (ce que j'appelle "la beauté de la science" !), ça passe mal. Nous avons beau nous décarcasser pour rendre le cours attractif, vivant... les étudiants accrochent peu, et nos cours magistraux ne sont pas aussi efficaces que ce que nous voudrions. Si nous voulons être plus efficaces dans notre enseignement, il nous faut changer. Il faut réussir à rendre les étudiants moins passifs, plus actifs, plus responsables de leur apprentissage, de leurs progrès. Pour cela, il me semble que le travail en équipe est incontournable, et que PEG est un bon moyen pour faire travailler les étudiants sur le cours, et faire en sorte qu'ils ne limitent pas leurs ambitions au "savoir-refaire". L'avantage, c'est que tout le monde y trouve son compte, enseignants comme étudiants. 


\section{AnNeXe : EXEMPle DE COURS PROBlÉMATISÉ}

\section{Calcul approché d'une dérivée, vitesse de convergence, impact des erreurs d'arrondi}

Il s'agit d'un cours d'analyse numérique, délivré à des élèves-ingénieurs (INSA de Toulouse), au niveau L2, qui ont obtenu le bac "STI", et donc ont peu le sens de l'abstraction. C'est un premier contact avec les méthodes approchées, la notion d'erreur de méthode et les techniques pour la majorer.

\subsection{Position du problème}

Dans certaines situations, on a besoin de calculer la valeur de la dérivée d'une fonction, alors que l'on ne peut expliciter la forme analytique de cette dérivée. C'est en particulier le cas lorsque l'on ne dispose que des valeurs numériques de la fonction (mesures par exemple). Il nous faut alors trouver un moyen pour avoir une valeur approchée (aussi approchée que possible !) de cette valeur que nous ne pouvons évaluer exactement... et, autant que possible, donner une majoration de l'écart entre la valeur "exacte", inconnue, et la valeur calculée (on pourra ainsi "garantir" une certaine valeur, ou du moins que la vraie valeur se trouve dans un intervalle précis) ; on parlera alors de "majoration d'erreur", de "calcul d'erreur", et éventuellement d' "intervalle de confiance".

Restons un peu matheux et expérimentaux à la fois (vous verrez, cela n'a rien d'incompatible !) : prenons une fonction analytique assez simple (nous commencerons par $f$ définie par $\forall x \in \mathbb{R}_{+}^{*}, f(x)=2 \sqrt{x}$ ), dont nous connaissons la dérivée (n'hésitez pas $! f^{\prime}(x)=\ldots$ ), et donc la valeur de celle-ci en tout $x$, et calculons numériquement la quantité $g_{h}(x)=\frac{f(x+h)-f(x)}{h}$ pour différents $h$, de plus en plus petits, et par exemple $x=1$. Comme nous sommes (surtout?) intéressés par l'erreur, on calculera aussi $e_{h}(x)=\left|g_{h}(x)-f^{\prime}(x)\right|$. Vous pourrez bien sûr, par la suite, tester d'autres fonctions et d'autres valeurs de $x$.

\subsection{Travail informatique}

Intéressons-nous donc à l'évolution de cette erreur en fonction de $h$ : faites le graphe (Excel et Matlab) de cette évolution (vous pourrez utiliser ce que vous avez fait la semaine dernière pour $\frac{(1+h)-1}{h}$ pour des valeurs de $h$ de plus en plus petites: 1 , puis $\frac{1}{a}$, puis $\frac{1}{a^{2}}$, puis $\frac{1}{a^{3}}$, puis ...). Utilisez des coordonnées logarithmiques (instruction Matlab loglog au lieu de plot).

\subsection{Travail en équipe}

Travaillez maintenant en groupes de 4 (nous les appellerons "équipes"). Vous pouvez cependant, si vous le souhaitez, aborder la mise en œuvre informatique en travail personnel (c'est-à-dire le paragraphe 5.4), en utilisant les formules indiquées dans ce paragraphe 5.3.

Tout d'abord interprétez le graphe précédent en donnant une expression expérimentale de l'erreur.

Il s'agit maintenant de majorer l' "erreur de méthode", c'est-à-dire l'erreur (théorique) commise en calculant $g_{h}(x)$ à la place de $f^{\prime}(x)$. Pour cela, faites un développement limité (reste de Taylor) de $f(x+h)$ à l'ordre 2 , et déduisez-en qu'il existe $\theta \in] 0 . .1\left[\right.$ tel que $g_{h}(x)=f^{\prime}(x)+(h / 2) f^{\prime \prime}(x+\theta h) \mid$, donc $\left|g_{h}(x)-f^{\prime}(x)\right| \leq(h / 2) M_{2}$ où $M_{2}$ est un majorant de $\left|f^{\prime \prime}(u)\right|$ pour $u$ compris entre $x$ et $x+h$. Voilà qui constitue une majoration de l'erreur commise en prenant $g_{h}(x)$ à la place de $f^{\prime}(x)$. Remarquez que ce majorant est en $\mathcal{O}(h)$ (c'est à dire en gros "quelques fois $h$ "), et dépend de $M_{2}$.

Examinez maintenant l'impact d'une erreur commise sur la valeur évaluée de $f(x)$ et de $f(x+h)$. Soit $M$ un majorant de cette erreur. Remarquez au passage que cette erreur peut être liée à une erreur de mesure (si les valeurs de $f$ sont consécutives à une mesure), ou bien dues à l' "erreur d'arrondi", ou "erreur numérique" (c'est en particulier le cas pour $2 \sqrt{x}$ et les expérimentations proposées dans ce TP). Remarquez de plus que quelle que soit son origine, cette erreur ne saurait être inférieure à $M_{0} \varepsilon$ où $\varepsilon$ est la précision machine $\left(10^{-17}\right.$ sous Excel, ou Matlab en précision "longue", $10^{-7}$ sous Matlab en précision courte) et $M_{0}$ un majorant de $|f(x)|$ et $|f(x+h)|$. Majorez l'impact sur $g_{h}(x)$ de l'erreur commise sur la valeur évaluée de $f(x)$ et $f(x+h)$ par $\frac{2 M}{h}$, ou par $\frac{2 M_{0}}{h}$ selon l'origine de l'erreur. Que pensez-vous de l'erreur globale (regardez : c'est la somme de l'erreur de méthode 
et de l'erreur d'arrondi) ? Déduisez-en une majoration de cette erreur globale (la somme des majorations de l'erreur de méthode et des erreurs d'arrondi). Montrez qu'il y a un minimum, que vous calculerez. Tout ceci est-il cohérent avec les graphes obtenus en 5.2 ?

Trouvons maintenant une autre approximation de la dérivée de $f$ en $x$. Pour cela, faites un développement limité (reste de Taylor) de $f(x+h)$ à l'ordre 3 , faites de même pour $f(x-h)$ et déduisez-en une formule approchée de $f^{\prime}(x) \ldots$ Faites alors un graphe qui devrait vous convaincre de la justesse de votre formule. Majorez alors l'erreur de méthode (vous pouvez trouver $\frac{h^{2}}{6} M_{3}$ où $M_{3}$ est un majorant de $\left|f^{\prime \prime \prime}(u)\right|$, pour $u$ compris entre $x-h$ et $x+h)$. Majorez maintenant l'impact d'une erreur commise sur la valeur évaluée de $f(x-h)$ et $f(x+h)$, enfin l'erreur totale. Comme précédemment, montrez que ce majorant admet un minimum, que vous calculerez.

On peut faire encore mieux ! Faites un développement limité (reste de Taylor) de $f(x+2 h)$, de $f(x+h)$, de $f(x-h)$ et de $f(x-2 h)$, à l'ordre 5 , et déduisez-en une formule approchée pour $f^{\prime}(x)$ (vous devez trouver $\left.\frac{-f(x+2 h)+8 f(x+h)-8 f(x-h)+f(x-2 h)}{12 h}\right)$, et majorez l'erreur de méthode (vous pouvez trouver $\frac{h^{4}}{30} M_{5}$ où $M_{5}$ est un majorant de $\left|f^{\prime \prime \prime \prime \prime}(u)\right|$, pour $u$ compris entre $x-2 h$ et $\left.x+2 h\right)$. Donnez pour cette formule un majorant de l'erreur d'arrondi (vous pouvez trouver $\frac{18 M_{0}}{12 h}$ où $M_{0}$ est un majorant de $|f(x-2 h)|,|f(x-h)|,|f(x+h)|$ et $|f(x+2 h)|)$. Là encore, montrez que l'erreur globale est majorée par un terme qui admet un minimum, que vous calculerez.

Discutez sur l'intérêt de ces différentes approximations.

\subsection{Travail informatique}

Tracez sous Excel et sous Matlab les erreurs expérimentales (sur un même graphe).

En groupe (mais vous pouvez avoir réfléchi à la question en travail personnel), interprétez les pentes, localisez les minima, et comparez avec l'étude théorique que vous avez faite en 5.3.

Vous pouvez enfin reprendre les expérimentations numériques avec d'autres fonctions et valeurs de $x$.

\subsection{A l'issue de ce travail, vous devez}

Avoir compris comment lire un graphe en coordonnées logarithmiques,

En particulier savoir exprimer l'équation d'une fonction représentée par une droite,

Avoir compris le principe du calcul d'une valeur approchée et du calcul d'erreur,

Savoir que souvent l'erreur d'arrondi augmente lorsque l'erreur de méthode diminue, de sorte qu'il y a présence d'une erreur minimale,

Savoir qu'en changeant de méthode il est quelquefois (souvent !) possible d'améliorer le résultat,

Avoir fait une première approche de la notion de vitesse de convergence,

Avoir une première approche de l'intérêt des développements limités pour déterminer une formule d'évaluation approchée et pour le calcul d'erreur,

... Savoir de mieux en mieux manipuler Excel et Matlab !

\section{BiBliogRAPHIE}

[1] R. V. Krivickas, Active Learning at Kaunas University of Technology, Global Journal of Engineering Education, Vol. 9, n¹, 2005 (UICEE)

[2] J. Proulx, Apprentissage par projet. 2004. Presses de l'université du Québec

[3] P. Sobieski, A.M.Trullemans, L. Warnier, B. Raucent, « Projets interdisciplinaires pour l'intégration progressive des compétences au long d'un curriculum polytechnique, exemple d'implémentation en $1^{\circ}$ cycle $\gg$, Louvainla-Neuve, 2008.

Pour en savoir plus sur PEG : http ://enseignants.insa-toulouse.fr/fr/ameliorer mon cours/peg.html

Pour en savoir plus sur l'APP : http ://www.apsq.org/sautquantique/doss/d-app.html ;

http://www.uclouvain.be/362060.html ; http://edutechwiki.unige.ch/fr/Apprentissage_par_problèmes

http ://edutechwiki.unige.ch/fr/Apprentissage_par_projet ; 